\title{
QUELQUES REFLEXIONS SUR LE CONCEPT D'ENTROPIE ISSUES D'UN ENSEIGNEMENT DE THERMODYNAMIQUE
}

\author{
BROSSEAU, C. ${ }^{1}$ et VIARD, J. ${ }^{2}$ \\ 1. Laboratoire de Spectrométrie Physique (Cermo), Université Joseph Fourier, B. P. 87, 38042 Saint- Martin- \\ d'Hères Cedex, France. \\ 2. 1. U. F. M., Université Joseph Fourier, B. P. 53 X, 38041 Grenoble Cedex, France.
}

\section{SUMMARY}

The authors establish the existence of a gap between physics' students conceptions concerning the notion of entropy and Clausius's, Boltzmann's, constructors of this concept. They analyze some basic features at the origin of these conceptions and some characteristics of the common sense interpretation of entropy. They conclude by some didactical propositions.

\section{INTRODUCTION}

Dans une première partie, nous analysons le concept d'entropie à travers les conceptions de Clausius et Boltzmann. Dans une seconde partie, à partir des résultats d'une enquête réalisée auprès d'un groupe d'étudiants, nous constatons un décalage entre les conceptions de ces étudiants et celles des pères fondateurs sur le contenu sémantique de la notion d'entropie. Enfin, en remarquant que le modèle de sens commun n'est ni dépourvu de pertinence ni de qualités, nous formulons quelques propositions pour l'enseignement.

\section{LA SEMANTIQUE DE L'ENTROPIE CHEZ CLAUSIUS ET BOLTZMANN}

\subsection{La contribution de Clausius (1868)}

II distingue: une première partie de lentropie liée à «' arrangement des molécules» à leur distance moyenne; elle «ne dépend pas de la température». Une autre partie au contraire «ne dépend pas de l'arrangement des molécules mais seulement de la température et de la quantité de chaleur contenue dans le corps» (chaleur est à prendre ici dans le sens d'énergie thermique). La première a un caractère intujtif évident, et Clausius lui donne le nom très évocateur de «disgrégation »: cette quantité mesure «le degré de division dù corps». L'action de la chaleur, fait remarquer Clausius, «tend à augmenter la disgrégation». Cette remarque fondamentale lui sert à définir une mesure de la disgrégation: "Comme l'accroissement de disgrégation est le moyen par lequel la chaleur effectue du travail, la quantité de travail doit être dans un rapport déterminé avec l'accroissement de disgrégation; nous fixerons la mesure, jusqu'à présent arbitraire, de celle$\mathrm{ci}$, de telle sorte qu’à une température donnée, I'accroisssement de disgrégation soit proportionnel au travail que la chaleur peut effectuer par son moyen».

La deuxième composante de l' entropie garde chez Clausius un caractère beaucoup plus abstrait, voire obscur. L'entropie totale est la somme de ces deux quantités qui peuvent varier indépendamment l'une de l'autre et dont les variations peuvent se compenser exactement de façon à assurer l'invariance de leur somme dans le cas où il n'y a pas d'apport de chaleur de l'extérieur. Il est important de remarquer que Clausius ne donne pas de signification physique à cette composante de l'entropie lié à la «chaleur contenue dans le corps». L'idée essentielle est, selon Iui, que la chaleur est un mouvement de particules; I'idée que ces particules en mouvement puissent avoir des vitesses différentes et que cette information sur la répartition des vitesses puisse être reliée à une partie de l'entropie est totalement absente. Par ailleurs, la température n'a pas de signification microscopique, elle n'est perçue 
que comme la mesure de la force ou de l'énergie contenue dans la chaleur.

\subsection{La contribution de Boltzmann (1902)}

C'est Boltzmann qui donne un sens physique à la deuxième partie de I'entropie en s'intéressant à la distribution des vitesses des molécules conséquemment aux travaux de Maxwell. La deuxième partie de l'entropie est reliée (en paraphrasant Clausius) à l'arrangement des vitesses des molécules dans l'espace des vitesses et non à l'arrangement des molécules dans l'espace «réel». Cet espace des vitesses est construit par Boltzmann dans une stricte analogie avec l'espace réel: ainsi à chaque instant la vitesse d'une molécule est représentée par ce qu'il appelle un "point vitesse» obtenu en portant à partir d'une origine arbitraire une parallèle au vecteur vitesse instantané de la molécule.

L'idée de cette représentation géométrique de l'état cinétique du gaz étant acquise, Boltzmann s'intéresse ensuite à la topologie de cet espace ou plutôt à l'une de ses reconstructions possibles: iI transforme son espace continu en un espace discret constitué de cellules distinctes de volumes égaux. Construire un état cinétique du gaz revient alors à attribuer, pour un volume de gaz donné, à la cellule $n^{\circ} 1, n_{1}$ points vitesse, à la cellule $n^{\circ} 2$, $\mathrm{n}_{2}$ points vitesse et ainsi de suite...

Cette répartition qui caractérise un état cinétique donné peut être construite de multiples façons. D'un point de vue théorique, cette construction se ramène au problème du tirage de boules dans une urne contenant un grand nombre de boules identiques mais de couleur différente (ex: $n_{1}$ boules rouges, $n_{2}$ boules bleues ...). Le dénombrement des différents «états» du gaz et par suite de la probabilité pour cet état de se réaliser, est donc accessible par des raisonnements d'analyse combinatoire. Boltzmann remarque snsuite que le logarithme du nombre précédent n'est autre (au signe près et à une constante dimensionnelle près) que sa quantité $\mathrm{H}$ dont le minimum permet, en rajoutant un terme prenant en compte le volume total du gaz, de retrouver l'expression donnée par Clausius de l'entropie du gaz parfait. L'analogie entre les deux composantes de l'entropie peut être enrichie par la remarque suivante: si la disgrégation est une fonction croissante de l'espace accessible aux molécules (donc du volume qu'elles occupent) l'entropie cinétique sera une fonction croissante de l'intervalle de vitesse offert aux molécules. L'espace accessible étant mesuré par le volume géométrique, nous observons que la température est une mesure de l'intervalle de vitesse accessible. Ce fait est bien visualisé par le graphe d'une distribution type Maxwell-Boltzmann où la température décrit la vitesse maximale des molécules. De ce fait la température acquiert avec les travaux successifs de Maxwell et Boltzmann une signification microscopique: elle caractérise la forme d'une distribution de vitesses, l'étalement de cette distribution. D'autre part, le fondement sémantique de la dépendance de l'entropie à l'égard de la température est élucidé: l'accroissement de la température, toutes choses étant égales par ailleurs, accroît l'intervalle des vitesses accessibles.

\subsection{Le réseau conceptueI}

Il est important de retenir finalement la mise en place progressive dans le temps grâce aux travaux de Clausius, Maxwell et Boltzmann d'un réseau conceptuel qui permet d'appréhender la notion d'entropie par les multiples relations qu'elle entretient avec les autres notions fondamentales.

Une idée centrale assure la cohérence de ce réseau conceptuel: la fonction essentielle du concept d'entropie est de caractériser une transformation thermodynamique affectant un système physique (dans l'exemple précédent un $g a z$ ), indépendamment de son aspect énergétique.

Cette intention est très nette chez Clausius puisqu'avant d'avoir construit le terme d'entropie à partir du préfixe 'en' (tiré d'énergie) et du mot grec tropê (qui signifie transformation), il désigne cette notion par la périphrase suivante: le «contenu de transformation du corps», de même qu'il désigne l'énergie par la périphrase «le contenu de chaleur et d'cuvrem.

Or quelle est la nature de cette transformation thermodynamique subie par le système physique si elle n'est pas d'ordre énergétique? Elle est essentiellement informationnelle ou structurelle, et relative non à la quantité d'énergie disponible mais à la «qualité» de cette énergie liée à sa répartition entre une collection d'un grand nombre d'éléments discrets.

L'information nécessaire à la caractérisation d'un système physique aussi complexe qu' un gaz comporte en dehors du grand nombre d'éléments (typiquement de l'ordre de $10^{23}$ ), deux constituants fondamentaux relatifs I'un à la position des éléments dans l'espace et l'autre à leurs vitesses.

Il est utile de donner une représentation de ce réseau conceptuel au moyen đu schéma suivant:

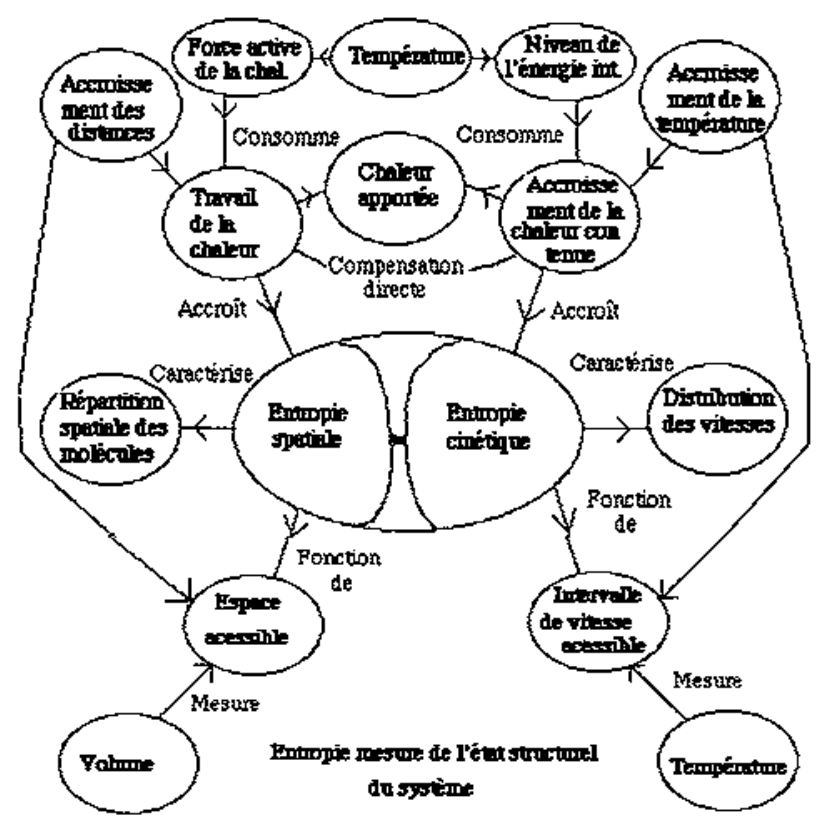




\section{LES CONCEPTIONS DES ÉTUDIANTS SUR L'ENTROPIE}

\section{1. Une enquête sous forme d'interview révèle un décalage important}

L'enquête réalisée par I'un des auteurs (Viard 1988) auprès d'un groupe d'une dizaine d'étudiants de D.E.A. de physique ou "thésarđs»" en physique, est centrée sur la situation problème suivante: on considère un gaz isolé thermiquement qui se détend de façon réversible. Que devient l'entropie du gaz dans cette transformation?

On a au préalable demandé une définition de l'entropie et également de préciser les relations de cette grandeur avec les autres paramètres du problème que sont la chaleur et la température. Le constat le plus important est que la quasi-totalité des étudiants interrogés ne possèdent pas les outils conceptuels. nécessaires à la résolution de ce problème.

La maîtrise du formalismen'est pas en cause: 7 interviewés sur 10 ont mentionné leur connaissance de la relation $\mathrm{dS}=\mathrm{dQ} / \mathrm{T}$ déterminant la dépendance de la variation de l'entropie à l'égard de la quantité de chaleur échangée par le système (nulle dans le cas présent).

Pourtant un seul parmi les dix, conclura à partir du constat d'absence d'échange thermique, au moyen de la relation précédente, à la constance de l'entropie. Le raisonnement dominant (7 cas sur 10) est le suivant: «L'entropie c'est le désordre ou la mesure du désordre; si le volume augmente, le désordre augmente, donc l'entropie augmente.»

\subsection{L'interprétation de cet échec}

Dans l'affirmation: «l'entropie reste constante», Ie raisonnement n'est pas en cause; c'est la sémantique, la signification attribuée à l'entropie: «le désordre spatial» qui entraîne la conclusion erronée. Il s'agit d'une erreur du contenu conceptuel. Nous suggérons qu'il y a, par l'enseignement, non pas transmission du contenu conceptuel, («le sens d'un message n'est pas dans le message" comme le remarque Lo Jacomo (1986), ainsi nous ne devrions pas parler de transmission de connaissance Iorsqu'il s'agit d'intelligibilité), mais qu'il s'établit une reconstruction du concept sur une base différente et incomplète. La base de référence n'est plus la description modéliśsée de l'état physique d'un gaz mais l'experience commune de la perte d'information relative à la localisation d'une collection d'objets lorsque l'espace englobant ces objets s'accroît. A partir de cette référence nouvelle le symbole 'entropie' va se charger de sens et être réellement appropriée par l'étudiant. Cette appropriation s'accompagne d'unchangement de dénomination d'entropie en désordre. L'étudiant se réfère ainsi au «désordre» pour faire fonctionner ce concept lors de la résolution d'un problème ("Quand le volume s'accroît, le désordre croît.»).

\subsection{Quelques traits de la conception de sens commun} de l'entropie

Il y a troncature du contenu conceptuel: I'entropie cinétique de Boltzmann est éliminée. La cohérence du réseau conceptuel que constitue l'entropie, la chaleur, la température, I'espace accessible est cependant maintenu au prix d'une réorganisation autour de la seule idée $d u$ désordre spatial qui se substitue à celle plus riche et plus complexe de perte d' information sur l'état physique ou de destructuration du système.

Cette réorganisation se fait au prix d'une confusion entre les rôles respectifs de la chaleur et de la température dans l'accroissement de I'entropie spatiale: «Plus c'est chaud (plus la température est élevée), plus il y a de désordre." alors que c'est l'apport de chaleur qui peut se faire sans élévation de température et non l'élévation de température qui accroît l'entropie spatiale. «Il y a de plus stricte séparation entre les notions de travail et de chaleur (négation du premier principe), par suite l'absence de chaleur échangée se traduit par la constance de la température, de même l'apport de chaleur par un accroissement de la température.

2.4. Le graphe conceptuel du modèle de sens commun (à comparer avec le précédent)

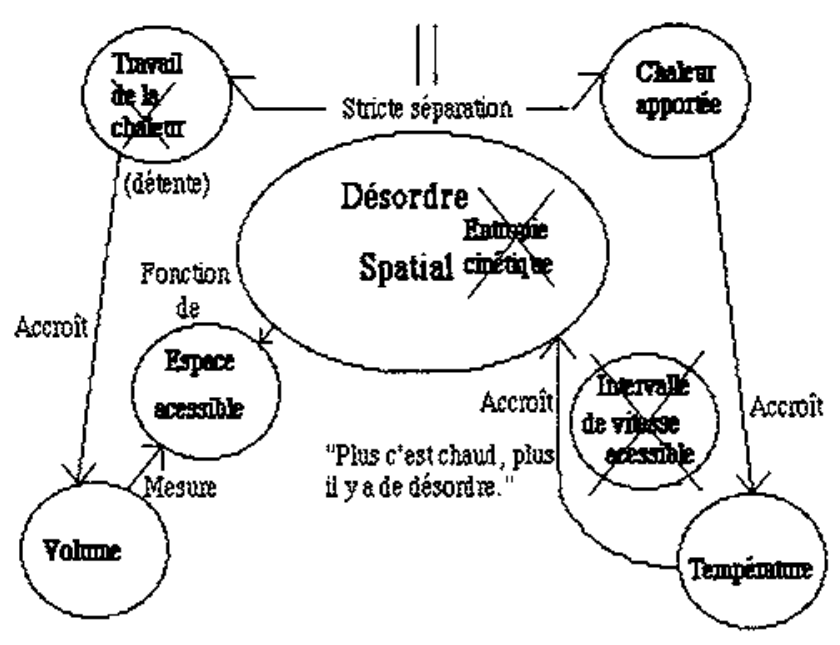

\section{LA PERTINENCE DUMODÈLE.QUELQUES PROPOSITIONS DIDACTIQUES}

\subsection{La pertinence du modèle}

Le modèle fonctionne de façon satisfaisante dans deux transformations importantes: la détente isotherme ou le chauffage du gaz à volume constant, dans ces deux cas le modèle de sens commun rend parfaitement compte de l'accroissement de l'entropie par l'accroissement du volume dans un cas et par celui de la température dans l'autre.

Par contre, dans le cas de la détente adiabatique (objet du problème proposé dans cette interview) où le volume 
et la température varient simultanément, ce modèle ne permet plus de prévoir l'évolution de l'entropie. Or, curieusement, nous remarquons que cette transformation plus complexe est rarement traitée dans le détail, à la différence des deux précédentes, dans les ouvrages de référence en thermodynamique bien qu'elle soit, comme nous venons de le voir, apte à départager les deux modèles.

Il est également pertinent de remarquer que ce modèle de sens commun présente une grande simplicité tout en assurant l'unification entre les approches thermodynamique et statistique. Ces avantages joints à la faculté de rendre compte de l'évolution de l'entropie dans les deux situations classiques rapportées plus haut lui confèrent des atouts non négligeableset peuvent foumir unélément d'explication de sa résistance à plusieurs années d'enseignement.

\subsection{Propositions didactiques}

Nous proposons d'introduire une composante de rétroaction en instaurant une situation conflictuelle révélatrice de l'écart entre le concept initial que le physicien prétend développer et celui qui reste après enseignement, la mesure de cet écart fournissant un critère d'efficacité de l'enseignement ainsi que des indications pour ses modifications ultérieures.

\section{REFERENCES BIBLIOGRAPHIQUES}

BACHELARD, G., 1981. Essai sur la connaissance approchée. (Jean Vrin: Paris).

BOLTZMANN, L., 1902. Leçons sur la théorie des gaz. (GauthierVillars: Paris).

CLAUSIUS, R., 1868. Théorie Mécanique de lachaleur. (Eugène Lacroix: Paris).
Pour répondre à l'objection de banalité qui pourrait nous être faite, nous remarquons que l'acquisition des concepts est rarement évaluée. L'évaluation pratiquée, en cours, ou en fin d'enseignement, se limite le plus souvent à des tests de pratiques opératoires à partir de symboles formels. La seule utilisation du formalisme n'implique pas la mobilisation du concept et il arrive souvent dans ce genre d'exercice que, comme le souligne Bachelard (1981), "le signe (formel) prime la chose signifiée» (i.e. Ie concept physique) au point de l'évacuer entièrement.

L'évaluation que nous proposons suppose au contraire de construire des situations où les concepts acquis devront nécessairement fonctionner sous un contrôle personnalisé et autonome de leur utilisateur (il ne faut pas que l'étudiant soit guidé pas à pas dans la résolution mais incité à penser par lui même en physicien et par suite mobiliser ses conceptions). La difficulté technique et formelle du problème posé ne doit pas être trop élevée afin de permettel' autonomie desétudiants dans l'utilisation du concept. Mais ces situations doivent posséder une complexité conceptuelle suffisante pour permettre de départager les différents modèles ou conceptions. Il s'agit non pas de réduire la difficulté, mais de la déplacer du domaine de la technique vers celui de la sémantique. La situation problème de l'enquête précédente constitue en ce sens une tentative pour innover dans cette direction.

\section{RESUMEN}

El trabajo se ha dividido en tres partes: 1) análisis del concepto de entropía a través de las concepciones de Clausius y Boltzmann, 2) concepciones de los alumnos acerca de la entropía, y 3) reflexiones sobre el modelo detectado y proposiciones didacticas al respecto.

1. Siguiendo el itinerario de la entropía de Clausius a Boltzmann, los autores destacan la progresiva implantación de una trama conceptual que permite aprehender la noción de entropia a través de sus relaciones múltiples con las otras nociones fundamentales. Una idea central sostiene la red conceptual: la función esencial del concepto de entropía es la de caracterizar una transformación termodinámica que afecta a un sistema físico, independientemente de su aspecto energético.

2. Se han analizado los resultados de una encuesta realizada por uno de los autores a una decena de estudiantes de D.E.A. (equivale aproximadamente a los actuales Master) en física. La encuesta gira en torno a la evolucion de la entropía de un gas aislado que se expande de forma reversible.

La primera constatación es que la práctica totalidad de los estudiantes encuestados carecen del bagaje conceptual necesario para resolver el problema planteado.

La concepción que podemos llamar de "sentido común", que se manifiesta en el análisis de los resultados, se caracteriza por que en ella ha sido eliminada la entropía cinética de Boltzmann, con la ruptura consiguiente de contenido conceptual. Se mantiene, sin embargo, la coherencia de la trama conceptual que constituyen la entropía, el calor, la temperatura y el espacio accesible, pero ello ocurre al precio de una reorganización alrededor de la única idea del desorden espacial.

3. Los autores mencionan las transformaciones físicas en que el modelo funciona y aquélla en que falla, así como otras características del mismo que justifican su persistencia en el tiempo. Finalmente ofrecen sus propuestas didácticas basadas en la introducción de una componente de retroacción a través de la creación de una situación de conflicto capaz de revelar la brecha entre el concepto que el físico pretende desarrollar y el que permanece después de la enseñanza. El tamaño de esta brecha proporcionará un criterio sobre la eficacia de la enseñanza así como indicaciones para ulteriores modificaciones del proceso. 